\title{
A century-old debate on protein aggregation and neurodegeneration enters the clinic
}

\author{
Peter T. Lansbury ${ }^{1} \&$ Hilal A. Lashuel ${ }^{2}$
}

\begin{abstract}
The correlation between neurodegenerative disease and protein aggregation in the brain has long been recognized, but a causal relationship has not been unequivocally established, in part because a discrete pathogenic aggregate has not been identified. The complexity of these diseases and the dynamic nature of protein aggregation mean that, despite progress towards understanding aggregation, its relationship to disease is difficult to determine in the laboratory. Nevertheless, drug candidates that inhibit aggregation are now being tested in the clinic. These have the potential to slow the progression of Alzheimer's disease, Parkinson's disease and related disorders and could, if administered presymptomatically, drastically reduce the incidence of these diseases. The clinical trials could also settle the century-old debate about causality.
\end{abstract}

When Alois Alzheimer discovered that the postmortem brains of his severely demented patients contained proteinaceous amyloid plaques, he wondered whether these deposits caused or resulted from neurodegeneration ${ }^{1}$. A century later, this question has been vigorously investigated and debated, but not definitively answered. Protein aggregation of some type is now also known to be a characteristic feature of Parkinson's disease (PD), amyotrophic lateral sclerosis (ALS), Huntington's disease (HD) and many less prevalent neurodegenerative diseases. However, the fibrillar deposits that are found in postmortem diseased brains - for example, the amyloid plaques noted by Alzheimer, the Lewy bodies of PD and related diseases, and the nuclear inclusions of HD - can be present in the brains of asymptomatic individuals and do not correlate to the severity of disease at time of death. Spurred on by a greater understanding of the in vitro protein fibrillization process, a search for a specific neurotoxic prefibrillar aggregate and the pathway by which it causes neuronal dysfunction, death and disease is underway.

The precise relationship between protein aggregates such as the extracellular amyloid plaques and intracellular neurofibrillary tangles that characterize the Alzheimer's disease $(\mathrm{AD})$ brain and the neurodegeneration that produces symptoms has not been determined (Fig. 1). The possibility that protein aggregation causes neurodegeneration (as opposed to resulting from it) returned to centre stage with the discovery that a gene linked to autosomal dominant familial AD encodes a precursor (amyloid precursor protein, APP) of the amyloid- $\beta$ protein $(A \beta)^{2}$. A $\beta$ is a 4-kDa peptide that comprises the least soluble, fibrillar component of $\mathrm{AD}$ amyloid plaques ${ }^{3,4}$. During the past 15 years, a working hypothesis has been developed implicating protein aggregation as the trigger of the cascade of events that result in neurodegeneration and disease ${ }^{5}$. This model, which is known as the 'amyloid hypothesis', has evolved with our understanding of the process of protein aggregation and has withstood concerted efforts to disprove it. In recent years, the hypothesis has been generalized as it has become apparent that most neurodegenerative diseases are characterized by protein aggregates, albeit of varying composition. This review briefly summarizes the key experiments that led to the working hypothesis, but focuses on recent experimental findings that purport to test elements of the working model.
The amyloid hypothesis has been shaped by evidence drawn primarily from three disciplines: neuropathology, genetics and biophysics. Each class of evidence is powerful, but each also has limitations. Pathological studies have focused on defining and refining the correlation between protein deposits, neuronal dysfunction, neuronal loss and severity of symptoms at time of death. Because studies of human tissue rely on postmortem samples, it is difficult to glimpse the early stages of disease during which the triggering species is most likely to be observable. However, this critical period is theoretically observable in living patients by brain imaging. Recent advances towards the in vivo detection of

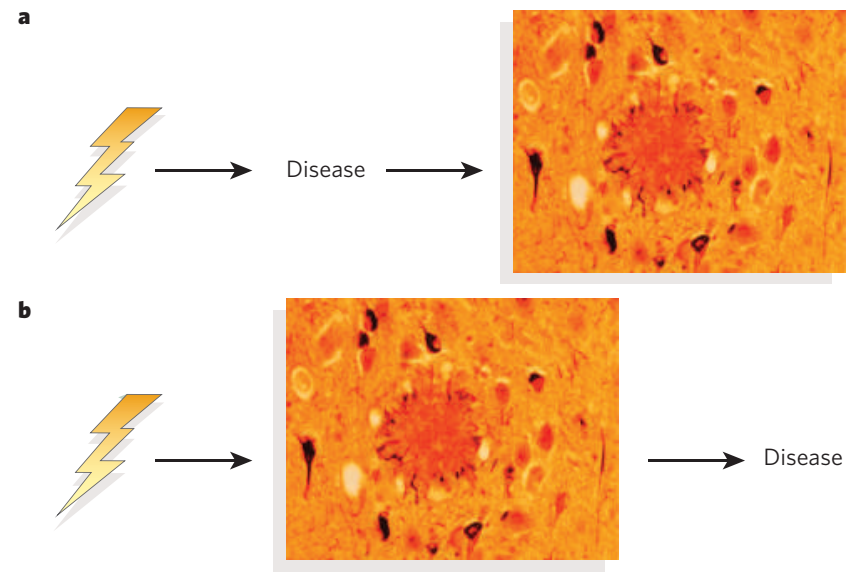

Figure 1 | Fibril formation and disease are linked. Two mutually exclusive hypotheses have been proposed to explain the correlation between neurodegenerative disease and fibrillar-protein deposits in the postmortem diseased brain. a, Hypothesis one: human neurodegenerative disease causes fibrillar deposits, but protein aggregation has no causal role. b, Hypothesis two: fibrillar protein deposits cause neurodegenerative disease. The tissue image is from an $\mathrm{AD}$ brain and shows an amyloid plaque surrounded by intraneuronal neurofibrillary tangles. 


\section{Box 1 | Imaging aggregates may allow presymptomatic diagnosis}

It has been estimated that neurodegeneration associated with $A D$ and PD starts 5 to 10 years before symptoms warrant a diagnosis. In the case of PD, imaging studies have shown that loss of $50 \%$ of the substantia nigra's dopaminergic neurons is tolerated; symptoms typically appear when $70 \%$ have been lost ${ }^{81}$. The development of methods to diagnose these diseases presymptomatically has not been a commercial priority, because available therapies target only the symptoms, not the underlying disease. However, this situation is about to change, as the use of therapies that slow the underlying disease (Box 2) will be greatly increased if disease can be diagnosed and treated presymptomatically (Fig. 4). As more genetic risk factors emerge, it will be possible to assess risk, but in order to justify treatment, it will be necessary to directly measure the disease process.

This is possible for PD, because the affected region of the brain is localized and excellent methods for imaging dopaminergic neurons are available. However, in the case of $A D$, direct imaging of protein aggregates may provide a more sensitive measure. William Klunk of the University of Pittsburgh has developed a positron emission tomography imaging reagent that targets fibrillar amyloid plaques and allows their detection in vivo ${ }^{6}$. However, given that fibrillar amyloid occurs relatively late in the disease process, it would also be useful to develop analogous reagents that target early $A \beta$-derived aggregates. A recent study in transgenic AD mouse models ${ }^{47}$ used classical biochemical separation methods to correlate a non-fibrillar $A \beta$-containing species $\left(A \beta^{\star} 56\right)$ with the appearance of an $A D$-like phenotype. An analogous study, correlating an early $A \beta$ aggregate with the first clinical signs of $A D$, would require the development of new technology and would have enormous practical consequences. A time can be imagined when individuals with a relative risk of $>2.0$, on the basis of genetic factors, would be routinely imaged at the age of 50 using such a reagent (Fig. 4). If abnormal $A \beta$ aggregates were present, treatment with a disease-modifying drug could begin immediately. This approach might effectively 'cure' many cases of AD.

amyloid plaques in human patients with $\mathrm{AD}^{6-8}$ mean that it may soon be possible to follow the natural history of protein aggregation and correlate it with disease progression (Box 1). In the meantime, studies of animal models have provided detailed information about certain phenotypes of the human disease ${ }^{9,10}$.

Genetic data are clearly relevant to human $\mathrm{AD}$ and have been instrumental in the building of a circumstantial case to support existing models of pathogenesis. The genes that have been linked to disease are predominantly of two types: familial and susceptibility factors. Several genes of the former type have been identified, and, when mutated, these cause rare, early-onset forms of disease. Examples include the genes encoding APP, which is involved in familial AD, and $\alpha$-synuclein, which is involved in familial PD. Whether these monogenic subtypes are representative of the vast majority of cases, which are believed to originate from a complex mixture of genetic and perhaps environmental factors, is debatable (see below). The latter type of disease-gene link is based on a susceptibility factor, such as apolipoprotein-E genotype in $\mathrm{AD}^{11}$ or $\mathrm{UCH}-\mathrm{L} 1$ genotype in $\mathrm{PD}^{12}$. Although these susceptibility factors are found in many patients, it is difficult to translate their subtle effects into a working hypothesis.

Finally, biophysical studies have been increasingly influential in this field owing to their ability to provide a mechanistic rationale to better explain the effects of disease-causing mutations - for example, why the $A \beta 42 / A \beta 40$ ratio is more critical than the total amount of $A \beta$ produced $^{13}$. The obvious shortcoming of such studies is that the idealized experimental systems that they require bear little resemblance to the human brain. Moreover, the pathological processes are difficult to study in vitro, because, unlike biologically necessary processes (such as the complement cascade) or structures (for example, the proteasome), protein aggregation has not been optimized by evolution and is difficult to reconstitute in an artificial environment (in fact, evolution has probably done its best to reduce protein aggregation).

This review discusses and evaluates a series of increasingly detailed hypotheses that seek to explain the relationship between protein aggregation and neurodegenerative disease. We focus on $\mathrm{AD}$, because it set the historical course for the development of the field. AD is also the most prevalent human neurodegenerative disease, and, for that reason, is the subject of intense drug-development efforts. However, examples from other neurodegenerative diseases are discussed where appropriate. We also examine the state-of-the-art working hypotheses, asking what can be done to refine and clarify the current models, and more importantly, what needs to be done in order to produce disease-modifying treatments for neurodegenerative diseases and to identify presymptomatic individuals who could benefit from such treatments.

\section{Fibrillar aggregates could be the cause or effect of disease}

The two early hypotheses (Fig. 1) in this field were predicated on the fact that fibrillar deposits were the only observable proteinaceous abnormalities in postmortem human tissue. The two are mutually exclusive, but do not, as explained below, encompass all possibilities: both could be wrong. Although they differ in terms of the causal relationship between protein fibrils and disease, both posit that fibrillar aggregates and disease are linked.

As the list of neurodegenerative diseases characterized by fibrillar aggregates grew ${ }^{14}$, it became apparent that subtypes of several such diseases are not characterized by the presence of fibrillar deposits in the brain. This finding indicates that fibrillar deposits are not generally coupled to disease and, therefore, that hypotheses one and two are both incorrect. It is possible that diseases that do not show fibrillar aggregation, which include Gerstmann-Straussler syndrome (a genetic prion disease $)^{15}$, autosomal recessive juvenile parkinsonism (AJPR, a juvenileonset form of PD) $)^{16}$ and sporadic $\mathrm{ALS}^{17}$, are not representative of most neurodegenerative diseases. However, the number and size of plaques in a postmortem $\mathrm{AD}$ brains do not correlate with the severity of symptoms at the time of death ${ }^{18,19}$, which also argues against hypotheses one and two. Finally, another fact that conflicts with the second hypothesis is that amyloid plaques are found throughout the cortex of many cognitively normal 70-year-olds ${ }^{20}$.

\section{Fibrillar aggregates are an epiphenomenon of disease}

So, a revised hypothesis is necessary to explain the qualitative, but not quantitative, correlation between fibrillar deposits and disease. This model (hypothesis number three, Fig. 2) allows for the extreme situations described above - prevalent deposits accompanied by little or no disease on the one hand and severe disease with little or no deposits on the other.
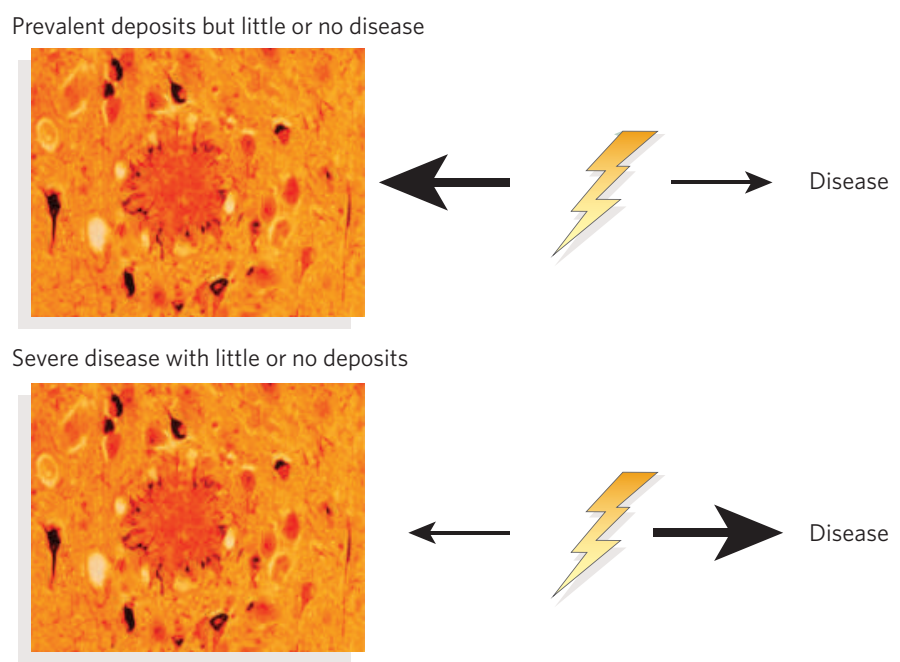

Figure 2 | Fibrillar deposits are not quantitatively correlated with disease. There is a qualitative, but not quantitative, correlation between fibrillar deposits and disease. One perturbation initiates both fibril formation and disease (lightning bolt), although the agent responsible for this is unknown. 
According to this hypothesis, many different events (the lighting bolts in Fig. 2) could cause both fibril formation and disease. However, given that the fibrillar protein aggregates arise from a normally expressed protein (such as $\mathrm{A} \beta$ or $\alpha$-synuclein) by a complex pathway, it is logical to start by postulating that a precursor to the fibrillar aggregates may be the triggering factor ${ }^{21}$. This hypothesis is supported by studies in animal models, which reinforce the conclusion that fibrillar deposits are not directly related to disease $\mathrm{e}^{22-24}$, and in some cases demonstrate that fibrillar deposits and disease-like phenotype are anticorrelated. Thus, perturbations that increase deposits decrease the disease-like phenotype and vice versa ${ }^{23,25,26}$. The in vitro discovery and characterization of prefibrillar protein aggregates provided a framework for this hypothesis.

\section{Protofibrils are early intermediates in fibril formation}

Although conventional light microscopic analysis of postmortem AD brains revealed only fibrillar protein deposits, immunostaining with antibodies to $A \beta$ allowed detection of another type of $A \beta$ deposit, known as diffuse amyloid ${ }^{27}$. This deposit lacks fibrillar substructure and was proposed to be a precursor to fibrillar amyloid. In contrast to the fibrillar deposits, which can be extracted from the brain because of their unusual stability, diffuse amyloid proved impossible to extract and characterize $e^{28}$. Therefore, the first non-fibrillar $A \beta$ aggregates to be characterized were produced in vitro, from synthetic $\mathrm{A} \beta$.

Two groups, using complementary methods, demonstrated that $\mathrm{A} \beta$ produces non-fibrillar aggregates, which are consumed as fibrils are formed $^{29,30}$. These species were designated protofibrils, because the time dependence of their formation and the disappearance of their secondary structure (primarily $\beta$-sheet) were consistent with their being an intermediate in the fibrillization process (whether or not protofibrils are on the direct pathway from monomers to fibrils is still unclear). Notably, both groups reported that $A \beta 42$ forms protofibrils more rapidly than $\mathrm{A} \beta 40$, which is consistent with the fact that $\mathrm{A} \beta 42$ is much more strongly correlated with the disease process.

Subsequently, other non-fibrillar A $\beta$ aggregates have been described, including $A \beta$-derived diffusible ligands (ADDLs) ${ }^{31}, A \beta$ amylospheroids $^{32}$, membrane-associated $A \beta$ oligomers and annular $A \beta$ protofibrils $^{33,34}$. How these species are related to one another is difficult to determine, because the conditions for their formation and characterization vary widely (Fig. 3). Furthermore, although it is likely that these metastable oligomeric species ${ }^{35}$ assemble in a stepwise process involving

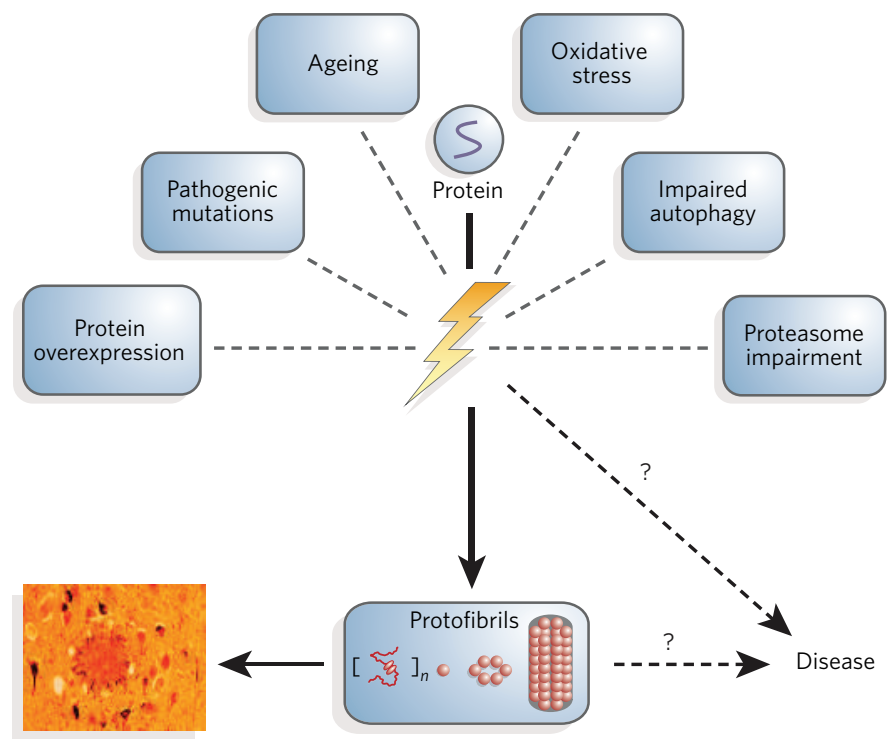

Figure 3 Disease and fibril formation may have a common cause. A number of factors have been proposed to trigger protein oligomerization (protofibril formation) and disease. Whether protofibrillar aggregates are the cause of disease is uncertain, but circumstantial evidence supports a pathogenic role for these structures.
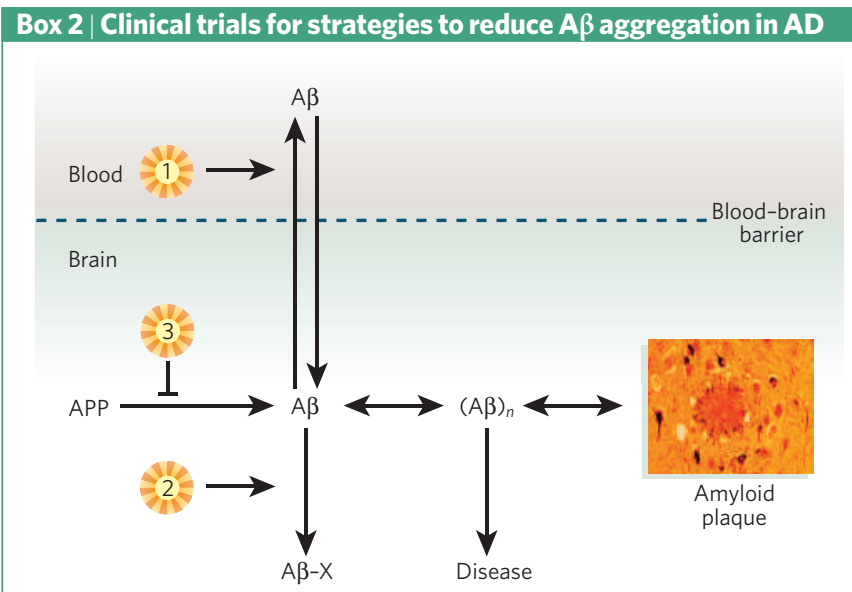

Three therapeutic strategies for Alzheimer's disease, all based on reducing the amount of protofibrillar $A \beta$ aggregation $\left((A \beta)_{n}\right)$, have reached phase II clinical trials. Each involves a different mechanism (see panel).

The first of these is Elan's $A \beta$ vaccine. Inoculation of mice with an $A \beta$-derived immunogen resulted in the reduction of amyloid plaques ${ }^{82}$ and a degree of rescue of some AD-like phenotypes ${ }^{24,83}$. In subsequent animal studies, it was shown that passive immunization, by infusion of $\mathrm{A} \beta$-directed antibodies, has a similar effect ${ }^{84}$, leading to the hypothesis that the vaccine causes redistribution of $A \beta$ from the brain into the periphery, where it can be degraded ${ }^{85}$ (1). A reduction in brain $A \beta$ would slow or even stop new aggregation, leading to a decrease in the rate of neurodegeneration. In addition, such a reduction could lead to complete resolution of amyloid plaques, which seem to be in dynamic equilibrium with monomeric $A \beta$ and thus may be actively cleared.

A second strategy (2) involves the inhibition of all $A \beta$ aggregation by binding monomeric $A \beta$ with a small molecule ( $X$; Neurochem's 'Alzhmed' (tramiprosate)) that is thought to be a proteoglycan mimic. This reduces amyloid-plaque formation in mouse models (and also rescues certain AD-like phenotypes) ${ }^{86}$. The compound is well-tolerated and can cross the blood-brain barrier. Phase II trials suggested that a subset of patients with mild AD experienced some improvement in cognitive measures after receiving treatment for more than 1 year (http://www.neurochem.com/ResearchActivities.htm). Phase III trials, the ultimate determinant of efficacy, are underway.

A third strategy (3) involves the reduction of $A \beta 42$ production from APP (and A $\beta 42$ aggregation), by modulating $\gamma$-secretase/presenilin activity. This strategy originated with the epidemiological finding that chronic arthritis patients had a lower incidence of $A D^{87}$. It was determined that the use of some, although not all, non-steroidal antiinflammatory drugs (NSAIDs) was responsible for this effect, and that the efficacy of NSAIDs did not correlate with their ability to inhibit cyclooxygenase $2(\mathrm{COX} 2)^{88}$. Myriad Genetics (http://www.myriad. com) is developing a compound, MPC-7869 ( $R$-flurbiprofen, known as 'Flurizan'), that is one component of the racemic drug Flurbiprofen (Ansaid) and is itself inactive with respect to COX2 inhibition. MPC7869 has been shown to reduce the levels of $A \beta 42$ in cell culture ${ }^{89}$ and in animal models of $A D^{90}$ by modulating the activity of $\gamma$-secretase, and is currently being evaluated in phase III clinical trials. It is hoped that this compound will not exhibit the side-effect profile associated with COX2 inhibitors. In a phase II study, patients with mild AD showed a decreased rate of decline in several clinical measures of $A D$ severity, although moderately affected patients showed no significant improvement. Several pharmaceutical companies have reported that $\gamma$-secretase inhibitors, which lower $A \beta 42$ and $A \beta 40$ by an active-site-directed mechanism, are entering phase II trials.

An alternative strategy to reduce aggregate levels involves the stimulation of aggregate degradation (possibly by autophagy). Finally, the idea that the promotion of fibril formation (at the expense, presumably, of protofibril) would be therapeutic is based on the aggresome proposal of Kopito ${ }^{91}$ and has shown some merit in a Drosophila model of $\mathrm{PD}^{25}$ and in cell culture. However, this strategy carries considerable risk, because fibrils themselves may be toxic ${ }^{71}$ 
low-molecular-weight oligomers, the relative importance of these is difficult to assess because they are too unstable to characterize ${ }^{36-38}$.

The intermediacy of stable, structured oligomers is a common feature of in vitro fibrillization by many disease-associated proteins, including a-synuclein ${ }^{39,40}$, huntingtin ${ }^{41}$ and islet amyloid polypeptide ${ }^{42,43}$, as well as by fibril-forming proteins that are unrelated to disease ${ }^{44}$. Non-fibrillar aggregates have also been isolated from cell culture ${ }^{45,46}$, from the brains of animal models of $\mathrm{AD}^{21,47}$ and from diseased human brains ${ }^{48-51}$. Annular pore-like aggregates have been observed during the in vitro fibrillogenesis of many well-characterized amyloid-forming proteins ${ }^{52}$. Annular a-synuclein-containing structures, similar in morphology to the structures formed in vitro, have been isolated from postmortem brain tissue of patients with multiple system atrophy (MSA) (ref. 51 and H.A.L., unpublished observations). MSA, like Parkinson's disease and diffuse Lewy-body disease, is characterized by $\alpha$-synuclein deposition in the form of cytoplasmic Lewy bodies.

\section{Fibrillar aggregates and disease may have a common cause}

A revised model of pathogenesis, which takes into account the complexity of the fibrillization process, states that one perturbation, or a combination of several (Fig. 3, lightning bolt), causes protofibril formation, fibril formation and disease. Whether fibril formation is caused by protofibril formation, as shown in Fig. 3, or merely co-occurs with protofibril formation is not as important, from a drug development perspective, as defining the relationship between protofibril formation and disease (dashed arrows). It is tempting to speculate that protofibril formation may cause both fibril formation and disease, but first, the possibility that protofibril formation and disease are caused by elevated levels of monomer protein must be considered (hypothesis number four; Fig. 3).

\section{Accumulation of protein monomers is not the cause}

Neurodegeneration and the expression levels of certain disease-associated proteins are clearly related. For example, triplication ${ }^{53}$ or duplication $^{54}$ of the $\alpha$-synuclein gene causes PD, and overexpression to a lesser extent due to a promoter polymorphism increases an individual's risk of $\mathrm{PD}^{55}$. This dependence is consistent with aggregation being important for disease, but is it possible that the monomeric protein independently causes both aggregation and disease (Fig. 3, hypothesis number four)?

Several facts indicate (but do not prove) that the monomeric proteins are non-toxic. First, the disease-linked proteins - such as a-synuclein and superoxide dismutase (SOD1, known to be involved in ALS) - are highly expressed in the normal brain. Second, the disease-linked proteins are not homologous at the level of primary sequence or native tertiary structure, making it difficult to imagine that this diverse group of monomeric proteins activates a common toxic mechanism. However, all of the aggregates are characterized by $\beta$-sheet secondary structure that may be responsible for shared mechanisms of toxicity. Third, many of the mutations that cause familial neurodegenerative diseases do not significantly alter the encoded protein's native structure, but reduce its stability and hence its steady-state level in vivo while accelerating its aggregation in vitro (this is well documented for $\mathrm{AD}^{56}, \mathrm{PD}^{39,57-59}$ and $\mathrm{ALS}^{60}$ ). For example, the level of mutant SOD1 in the blood of patients with familial ALS is considerably lower than the level of wild-type SOD1, which is inconsistent with the monomeric protein's being toxic (furthermore, the amount of mutant protein is inversely correlated with the aggressiveness of disease $)^{61}$. Fourth, cell death in neurodegenerative diseases exhibits stochastic behaviour and seems to be highly dependent on the level of protein - that is, small changes in expression level have a notable effect on disease onset and progression ${ }^{62}$. This nonlinear dependence is not consistent with the protein monomer's being the pathogenic species, but is easily explained by a protein-aggregation mechanism.

Finally, three features of monogenic neurodegenerative diseases are inconsistent with the fourth hypothesis. First, the age of symptom onset in Huntington's disease does not depend on the dosage of the abnormal gene (in other words whether it is heterozygous or homozygous) ${ }^{63}$. This finding is inconsistent with hypothesis number four, but can be explained

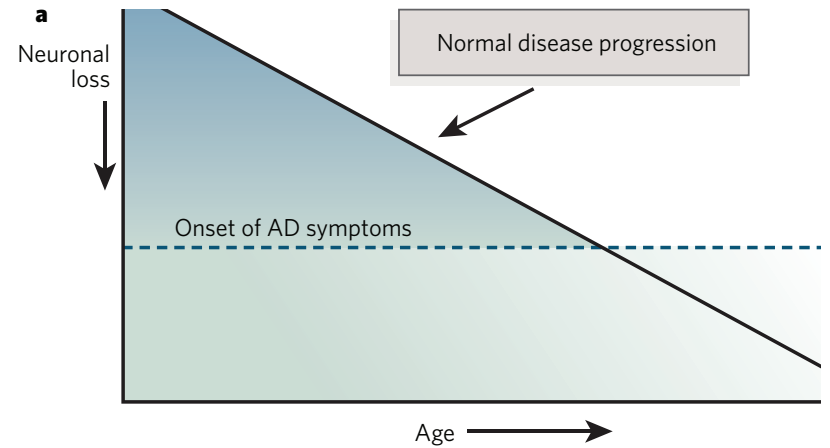

b

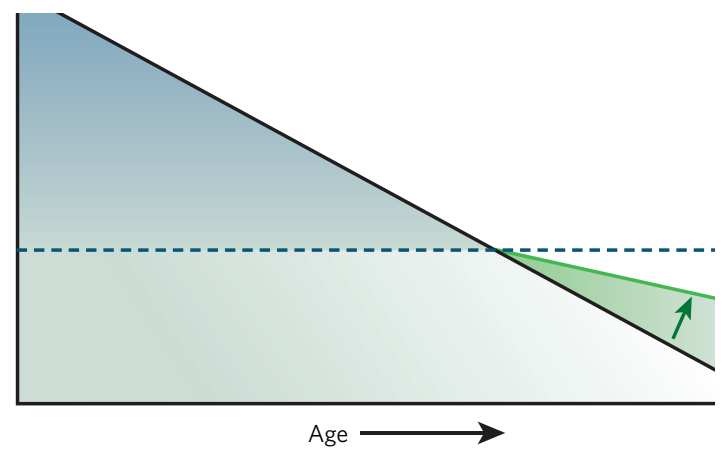

c

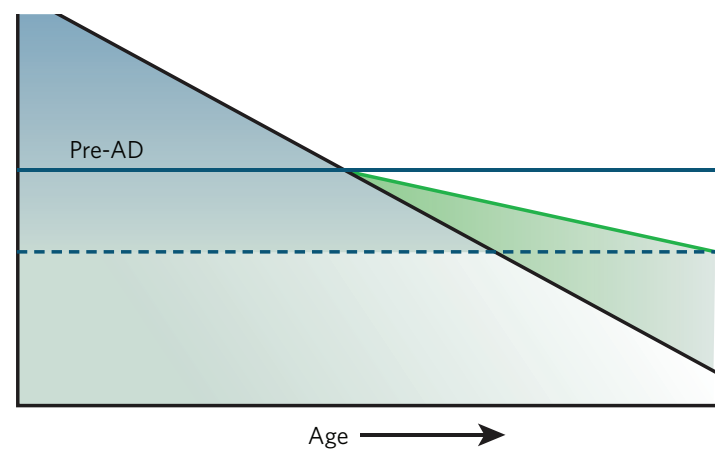

Figure 4 | Better diagnosis and presymptomatic treatment would drastically reduce the incidence and prevalence of neurodegenerative diseases. a, A diagram showing the loss of functional neurons over time that is characteristic of AD. Clinical diagnosis occurs when the disease is well underway, as symptoms do not become obvious until considerable neurodegeneration has occurred. b, A drug that slowed disease progression/neuronal loss, if started at clinical diagnosis, would have a significant effect on the impact of disease. $c$, If the same drug could be administered presymptomatically to patients diagnosed by, for example, imaging methods, the disease might not progress to the point at which symptoms become obvious.

by a seeded-polymerization model in which aggregation is nucleated by the product of the abnormal gene, and the aggregate can then grow equally well with either the mutant or the wild-type protein ${ }^{64}$.

Second, although an elevated level of plasma $A \beta 42$ is a risk factor for sporadic $\mathrm{AD}^{65}$, several presenilin mutations linked to familial $\mathrm{AD}$ do not affect $A \beta 42$ levels and decrease $A \beta 40$ production (however, most familial-AD-linked presenillin mutations increase $A \beta 42)^{66}$. This effect is inconsistent with any hypothesis contending that $\mathrm{A} \beta 42$ and/or $\mathrm{A} \beta 40$ elevation alone causes familial AD.

Third, the fourth hypothesis is difficult to reconcile with a phenomenon observed in sporadic prion disease ${ }^{67}$ and familial ALS ${ }^{68,69}$, whereby mutations or polymorphisms on separate alleles of the gene encoding the aggregating protein (the prion protein and SOD1, respectively) modulate the resultant disease. This conflicts with the idea of a protein monomer's being responsible for neurotoxicity, in which case the effects of two mutations should be additive. 
All of the data discussed above are consistent with the idea that nonfibrillar protein aggregates cause disease (hypothesis number five; Fig. 3). This hypothesis explains the lack of a gene-dosage effect in HD. It also explains why lowering $A \beta 40$ can cause familial $A D-A \beta 40$ inhibits the fibrillization of $A \beta 42$ (by stabilizing protofibrils) — and why raising the ratio of $A \beta 42$ to $A \beta 40$, whether by elevating $A \beta 42$ or by lowering $A \beta 40$, increases the rate of fibrillization ${ }^{70}$. Finally, the phenomenon of compound heterozygosity is consistent with the fifth hypothesis, because intermolecular interactions are critical for determining aggregation rate and disease onset and progression.

\section{Protofibrillar aggregates may trigger disease}

A demonstration that clinical status and the abundance of certain prefibrillar protein aggregates are not quantitatively correlated would disprove the fifth hypothesis. However, at present this experiment is not possible, because there are no available imaging methods capable of tracking the early stages of protein aggregation in vivo (Box 1). This hypothesis could also be disproved if a drug that could selectively inhibit $A \beta$ oligomerization and fibrillogenesis did not slow disease progression. In fact, three very different therapeutic strategies for $\mathrm{AD}$ that each seek to inhibit $\mathrm{A} \beta$ aggregation have shown some promise in reducing $\mathrm{AD}$ symptoms in phase II clinical trials (Box 2). It is important to note that the fifth hypothesis suggests that if fibrils are non-toxic (this is still an open question ${ }^{71}$ ), compounds that promote the conversion of early aggregates into fibrillar aggregates could slow disease progression.

\section{Several protofibrillar aggregates may be neurotoxic}

Because it is not currently possible to directly correlate the amount of a discrete protein aggregate to a human disease (Box 1), efforts have focused on cellular and mouse models of disease. Discrete protein aggregates have been identified both by in vitro aggregation of diseaseassociated proteins and in materials extracted from the brains of mouse models ${ }^{47}$ and human patients ${ }^{50,51}$. It is important to note that, in all of these, the extraction procedures and analysis methods (sodium dodecyl sulphate polyacrylamide gel electrophoresis, SDS-PAGE) are known to influence the dynamic aggregation process ${ }^{72,73}$. Thus, it is never clear whether the species being manipulated in the laboratory ever existed in the tissue of interest (in theory, imaging could clarify this; see Box 1). However, bearing this caveat in mind, several compelling correlations have recently been reported. In each of these, the monomeric protein does not affect the phenotype of interest (providing further evidence against the second hypothesis) and in many (but not all ${ }^{71}$ ) cases, the fibrillar aggregate also has no effect.

Non-fibrillar A $\beta$ preparations enriched in SDS-stable oligomers, spherical 4-6-nm aggregates and chain-like aggregates have all been shown to be toxic to cultured neurons, to inhibit hippocampal long-term potentiation (LTP $)^{31,32,36,74,75}$, to impair synaptic functions and to disrupt cognition and learned behaviour in rats $s^{47,76}$. Non-fibrillar $\alpha$-synuclein aggregates inhibit proteasomal activity ${ }^{77}$ and induce Golgi fragmentation $^{78}$. Furthermore, non-fibrillar aggregate preparations produced from several amyloid-forming proteins, regardless of differences in their size and/or morphological distribution, have been shown to be toxic to cultured cells/neurons, suggesting that a wide range of different aggregate species may be neurotoxic.

The problem with all of the correlative studies done so far is that they each reduce a complex human disease to a single measurable phenomenon - for example, 'the memory dysfunction in AD results from LTP deficit'. This oversimplification is required in order to reach clear conclusions, but $\mathrm{AD}$ (and PD, ALS and other similar disorders) probably results from a combination of biochemical, cell biological and systemic events (such as LTP deficit) each of which may, in turn, be influenced by one or more aggregate species. Ultimately, postmortem pathological data from human clinical trials of drug candidates that target specific processes (Box 2) will clarify the situation. However, in order to obtain this data, we must recognize that our current level of understanding, although incomplete, is sufficient for the development of such drug candidates.

\section{We can change the medical landscape}

It is critical to recognize that the onset of the neurodegenerative-disease process - which proceeds from the start of neuronal dysfunction - typically predates the first recognition of symptoms by at least 5-10 years (Box 1). Thus, if the presymptomatic disease could be diagnosed and treated, the prevalence of symptomatic disease could be greatly reduced (Fig. 4). Because most neurodegenerative diseases are genetically complex (HD, which is purely familial and monogenic, is the exception), presymptomatic diagnosis will require either the discovery of a peripheral marker for neurodegeneration that reacts earlier and is more sensitive than the traditional symptoms (for example, alteration of gene expression in the periphery), or that the process of protein aggregation be observable in the brain (Box 1). The development of useful imaging technology does not require that the relationship between aggregation and disease be explained in detail, only that a prefibrillar target be identified and that a target-specific probe exist. Once early protein aggregation has been detected, the patient will be treated with a disease-modifying drug designed to reduce the amount of aggregates in the brain. Once again, it would not be necessary to identify one specific target providing that the strategy used reduced all aggregation - for example, by reducing production of the aggregating protein (Box 2) or by stimulating aggregate clearance ${ }^{38,79,80}$. Ultimately, Alzheimer's 100 -year-old question, "what is the relationship between protein aggregation and neurodegeneration?" cannot be answered until the effects of such compounds on human disease are evaluated. The clinical trials will be large, time-consuming, risky and expensive, but we cannot afford to wait.

1. Alzheimer, A. Über eine eigenartige Erkrankung der Hirnrinde. Allg. Z. Psychiatr. Psychisch. Gerichtl. Med. 64, 146-148 (1907)

2. Goate, A. et al. Segregation of a missense mutation in the amyloid precursor protein gene with familial Alzheimer's disease. Nature 349, 704-706 (1991).

3. Glenner, G. G. \& Wong, C. W. Alzheimer's disease: initial report of the purification and characterization of a novel cerebrovascular amyloid protein. Biochem. Biophys. Res. Commun. 120, 885-890 (1984).

4. Masters, C. L. et al. Amyloid plaque core protein in Alzheimer disease and Down syndrome. Proc. Natl Acad. Sci. USA 82, 4245-4249 (1985).

5. Hardy, J. \& Selkoe, D. J. The amyloid hypothesis of Alzheimer's disease: progress and problems on the road to therapeutics. Science 297, 353-356 (2002).

6. Klunk, W. E. et al. Binding of the positron emission tomography tracer Pittsburgh compound-B reflects the amount of amyloid- $\beta$ in Alzheimer's disease brain but not in transgenic mouse brain. J. Neurosci. 25, 10598-10606 (2005).

7. Mathis, C. A., Klunk, W. E., Price, J. C. \& DeKosky, S. T. Imaging technology for neurodegenerative diseases: progress toward detection of specific pathologies. Arch. Neurol. 62, 196-200 (2005)

8. Fagan, A. M. et al. Inverse relation between in vivo amyloid imaging load and cerebrospinal fluid Aß42 in humans. Ann. Neurol. 59, 512-519 (2006).

9. Dodart, J. C., Mathis, C., Bales, K. R. \& Paul, S. M. Does my mouse have Alzheimer's disease? Genes Brain Behav. 1, 142-155 (2002).

10. Fernagut, P. O. \& Chesselet, M. F. Alpha-synuclein and transgenic mouse models. Neurobiol. Dis. 17, 123-130 (2004).

11. Roses, A. D. Apolipoprotein E alleles as risk factors in Alzheimer's disease. Annu. Rev. Med. 47, 387-400 (1996).

12. Satoh, J. \& Kuroda, Y. A polymorphic variation of serine to tyrosine at codon 18 in the ubiquitin C-terminal hydrolase-L1 gene is associated with a reduced risk of sporadic Parkinson's disease in a Japanese population. J. Neurol. Sci. 189, 113-117 (2001).

13. Jarrett, J. T. \& Lansbury, P. T. Seeding "one-dimensional crystallization" of amyloid: a pathogenic mechanism in Alzheimer's disease and scrapie? Cell 73, 1055-1058 (1993).

14. Ross, C. A.\& Poirier, M. A. Opinion: what is the role of protein aggregation in neurodegeneration? Nature Rev. Mol. Cell Biol. 6, 891-898 (2005).

15. Hsiao, K. \& Prusiner, S. B. Molecular genetics and transgenic model of GertsmannStraussler-Scheinker disease. Alzheimer Dis. Assoc. Disord. 5, 155-162 (1991).

16. Kitada, T. et al. Mutations in the parkin gene cause autosomal recessive juvenile parkinsonism. Nature 392, 605-608 (1998).

17. Rosen, A. D. Amyotrophic lateral sclerosis. Clinical features and prognosis. Arch. Neurol. 35, 638-642 (1978).

18. Terry, R. D. et al. Physical basis of cognitive alterations in Alzheimer's disease: synapse loss is the major correlate of cognitive impairment. Ann. Neurol. 30, 572-580 (1991).

19. Lemere, C. A et al. Sequence of deposition of heterogeneous amyloid $\beta$-peptides and APO E in Down syndrome: implications for initial events in amyloid plaque formation. Neurobiol. Dis. 3, 16-32 (1996).

20. Dickson, D. W. et al. Identification of normal and pathological aging in prospectively studied nondemented elderly humans. Neurobiol. Aging 13, 179-189 (1992).

21. Kuo, Y. M. et al. Comparative analysis of amyloid- $\beta$ chemical structure and amyloid plaque morphology of transgenic mouse and Alzheimer's disease brains. J. Biol. Chem. 276, 12991-12998 (2001).

22. Slow, E. J. et al. Absence of behavioral abnormalities and neurodegeneration in vivo despite widespread neuronal huntingtin inclusions. Proc. Natl Acad. Sci. USA 102, 11402-11407 (2005). 
23. Arrasate, M., Mitra, S., Schweitzer, E. S., Segal, M. R. \& Finkbeiner, S. Inclusion body formation reduces levels of mutant huntingtin and the risk of neuronal death. Nature 431 805-810 (2004)

24. Morgan, D. et al. A $\beta$ peptide vaccination prevents memory loss in an animal model of Alzheimer's disease. Nature 408, 982-985 (2000).

25. Chen, L.\& Feany, M. B. Alpha-synuclein phosphorylation controls neurotoxicity and inclusion formation in a Drosophila model of Parkinson disease. Nature Neurosci. 8, 657-663 (2005).

26. Bowman, A. B., Yoo, S. Y., Dantuma, N. P. \& Zoghbi, H. Y. Neuronal dysfunction in a polyglutamine disease model occurs in the absence of ubiquitin-proteasome system impairment and inversely correlates with the degree of nuclear inclusion formation. Hum Mol. Genet. 14, 679-691 (2005).

27. Nagy, Z. et al. The effects of additional pathology on the cognitive deficit in Alzheimer disease. J. Neuropathol. Exp. Neurol. 56, 165-170 (1997).

28. Roher, A. E. \& Kuo, Y. M. Isolation of amyloid deposits from brain. Methods Enzymol. 309, 58-67 (1999).

29. Harper, J. D., Wong, S. S., Lieber, C. M. \& Lansbury, P. T. Observation of metastable A $\beta$ amyloid protofibrils by atomic force microscopy. Chem. Biol. 4, 119-125 (1997).

30. Walsh, D. M., Lomakin, A., Benedek, G. B., Condron, M. M. \& Teplow, D. B. Amyloid $\beta$-protein fibrillogenesis. Detection of a protofibrillar intermediate. J. Biol. Chem. 272, 22364-22372 (1997)

31. Lambert, M. P. et al. Diffusible, nonfibrillar ligands derived from A $\beta 1-42$ are potent central nervous system neurotoxins. Proc. Natl Acad. Sci. USA 95, 6448-6453 (1998).

32. Hoshi, M. et al. Spherical aggregates of $\beta$-amyloid (amylospheroid) show high neurotoxicity and activate tau protein kinase $\mathrm{l} / \mathrm{glycogen}$ synthase kinase-3 $\beta$. Proc. Natl Acad. Sci. USA 100, 6370-6375 (2003).

33. Quist, A. et al. Amyloid ion channels: a common structural link for protein-misfolding disease. Proc. Nat/ Acad. Sci. USA 102, 10427-10432 (2005)

34. Lashuel, H. A., Hartley, D., Petre, B. M., Walz, T. \& Lansbury, P. T. Neurodegenerative disease: amyloid pores from pathogenic mutations. Nature 418, 291 (2002).

35. Lashuel, H. et al. Mixtures of wild-type and "Arctic" A 440 in vitro accumulate protofibrils, including amyloid pores. J. Mol. Biol. 332, 795-808 (2003).

36. Walsh, D. M. et al. Naturally secreted oligomers of amyloid $\beta$ protein potently inhibit hippocampal long-term potentiation in vivo. Nature 416, 535-539 (2002)

37. Townsend, M., Shankar, G. M., Mehta, T., Walsh, D. M. \& Selkoe, D. J. Effects of secreted oligomers of amyloid $\beta$-protein on hippocampal synaptic plasticity: a potent role for trimers. J. Physiol. (Lond.) 572, 477-492 (2006)

38. Cohen, E., Bieschke, J., Perciavalle, R. M., Kelly, J. W. \& Dillin, A. Opposing activities protect against age onset proteotoxicity. Science advance online publication, 10 August 2006 (doi:10.1126/science.1124646).

39. Conway, K. A. et al. Acceleration of oligomerization, not fibrillization, is a shared property of both $\alpha$-synuclein mutations linked to early-onset Parkinson's disease: implications for pathogenesis and therapy. Proc. Natl Acad. Sci. USA 97, 571-576 (2000).

40. Rochet, J. C., Conway, K. A. \& Lansbury, P. T. Inhibition of fibrillization and accumulation of prefibrillar oligomers in mixtures of human and mouse $\alpha$-synuclein. Biochemistry 39 , 10619-10626 (2000)

41. Poirier, M. A. et al. Huntingtin spheroids and protofibrils as precursors in polyglutamine fibrilization. J. Biol. Chem. 277, 41032-41037 (2002)

42. Anguiano, M., Nowak, R. J. \& Lansbury, P. T. Protofibrillar islet amyloid polypeptide permeabilizes synthetic vesicles by a pore-like mechanism that may be relevant to type II diabetes. Biochemistry 41, 11338-11343 (2002).

43. Green, J. D., Goldsbury, C., Kistler, J., Cooper, G. S. \& Aebi, U. Human amylin oligomer growth and fibril elongation define two distinct phases in amyloid formation. J. Biol. Chem. 279, 12206-12212 (2004).

44. Dobson, C. M. Protein folding and misfolding. Nature 426, 884-890 (2003).

45. Mukai, H. et al. Formation of morphologically similar globular aggregates from diverse aggregation-prone proteins in mammalian cells. Proc. Natl Acad. Sci. USA 102, 10887-10892 (2005)

46. Lee, H. J. \& Lee, S. J. Characterization of cytoplasmic $\alpha$-synuclein aggregates. Fibril formation is tightly linked to the inclusion-forming process in cells. J. Biol. Chem. 277, 48976-48983 (2002).

47. Lesne, S. et al. A specific amyloid- $\beta$ protein assembly in the brain impairs memory. Nature 440, 352-357 (2006).

48. Riesner, D. et al. Disruption of prion rods generates 10-nm spherical particles having high alpha-helical content and lacking scrapie infectivity. J. Virol. 70, 1714-1722 (1996).

49. Silveira, J. R. et al. The most infectious prion protein particles. Nature 437, 257-261 (2005).

50. Roher, A. E. et al. Morphology and toxicity of $A \beta-(1-42)$ dimer derived from neuritic and vascular amyloid deposits of Alzheimer's disease. J. Biol. Chem. 271, 20631-20635 (1996).

51. Pountney, D. L. et al. Annular $\alpha$-synuclein species from purified multiple system atrophy inclusions. J. Neurochem. 90, 502-512 (2004).

52. Lashuel, H. A. \& Lansbury, P. T. J. Are amyloid diseases caused by protein aggregates that mimic bacterial pore-forming toxins? Q. Rev. Biophys. advance online publication 18 September 2006 (doi:10.1017/50033583506004422).

53. Singleton, A. B. et al. a-Synuclein locus triplication causes Parkinson's disease. Science 302,841 (2003)

54. Chartier-Harlin, M. C. et al. Alpha-synuclein locus duplication as a cause of familial Parkinson's disease. Lancet 364, 1167-1169 (2004).

55. Maraganore, D. M. et al. Collaborative analysis of $\alpha$-synuclein gene promoter variability and Parkinson disease. J. Am. Med. Assoc. 296, 661-670 (2006)

56. Nilsberth, C. et al. The 'Arctic' APP mutation (E693G) causes Alzheimer's disease by enhanced A $\beta$ protofibril formation. Nature Neurosci. 4, 887-893 (2001).

57. Conway, K. A., Harper, J. D. \& Lansbury, P. T. Accelerated in vitro fibril formation by a mutant a-synuclein linked to early-onset Parkinson disease. Nature Med. 4, 1318-1320 (1998).

58. El-Agnaf, O. M. et al. Aggregates from mutant and wild-type $\alpha$-synuclein proteins and NAC peptide induce apoptotic cell death in human neuroblastoma cells by formation of beta-sheet and amyloid-like filaments. FEBS Lett. 440, $71-75$ (1998).
59. Greenbaum, E. A. et al. The E46K mutation in $\alpha$-synuclein increases amyloid fibril formation. J. Biol. Chem. 280, 7800-7807 (2005)

60. Hart, P. J. Pathogenic superoxide dismutase structure, folding, aggregation and turnover. Curr. Opin. Chem. Biol. 10, 131-138 (2006).

61. Sato, T. et al. Rapid disease progression correlates with instability of mutant SOD1 in familial ALS. Neurology 65, 1954-1957 (2005)

62. Perutz, M. F. \& Windle, A. H. Cause of neural death in neurodegenerative diseases attributable to expansion of glutamine repeats. Nature 412, 143-144 (2001).

63. Gusella, J. F. \& MacDonald, M. E. Molecular genetics: unmasking polyglutamine triggers in neurodegenerative disease. Nature Rev. Neurosci. 1, 109-115 (2000).

64. Lansbury, P. T. Structural neurology: are seeds at the root of neuronal degeneration? Neuron 19, 1151-1154 (1997)

65. Mayeux, R. et al. Plasma amyloid $\beta$-peptide 1-42 and incipient Alzheimer's disease. Ann Neurol. 46, 412-416 (1999).

66. Cupers, P. et al. The discrepancy between presenilin subcellular localization and $\gamma$-secretase processing of amyloid precursor protein. J. Cell Biol. 154, 731-740 (2001).

67. Parchi, P. et al. Molecular basis of phenotypic variability in sporadic Creutzfeldt-Jakob disease. Ann. Neurol. 39, 767-778 (1996).

68. Hand, C. K. et al. Compound heterozygous D90A and D96N SOD1 mutations in a recessive amyotrophic lateral sclerosis family. Ann. Neurol. 49, 267-271(2001).

69. Restagno, $G$. et al. The IVS1 $+319 t>a$ of SOD1 gene is not an ALS causing mutation Amyotroph. Lateral Scler. Other Motor Neuron Disord. 6, 45-49 (2005).

70. Hasegawa, K., Yamaguchi, I., Omata, S., Gejyo, F. \& Naiki, H. Interaction between $A \beta(1-42)$ and $A \beta(1-40)$ in Alzheimer's $\beta$-amyloid fibril formation in vitro. Biochemistry 38, 15514-15521 (1999).

71. Isaacs, A. M., Senn, D. B., Yuan, M., Shine, J. P. \& Yankner, B. A. Acceleration of amyloid $\beta$-peptide aggregation by physiological concentrations of calcium. J. Biol. Chem. advance online publication 26 July 2006 (doi:10.1074/jbc.M602061200)

72. Yu, W. et al. Oligomerization of amyloid $\beta$-protein occurs during the isolation of lipid rafts. J. Neurosci. Res. 80, 114-119 (2005).

73. Bitan, G., Fradinger, E. A., Spring, S. M. \& Teplow, D. B. Neurotoxic protein oligomers - what you see is not always what you get. Amyloid 12, 88-95 (2005).

74. Hartley, D. M. et al. Protofibrillar intermediates of amyloid $\beta$-protein induce acute electrophysiological changes and progressive neurotoxicity in cortical neurons. J. Neurosci. 19, 8876-8884 (1999).

75. White, J. A., Manelli, A. M., Holmberg, K. H., Van Eldik, L. J. \& Ladu, M. J. Differential effects of oligomeric and fibrillar amyloid- $\beta 1-42$ on astrocyte-mediated inflammation. Neurobiol. Dis. 18, 459-465 (2005)

76. Cleary, J. P. et al. Natural oligomers of the amyloid- $\beta$ protein specifically disrupt cognitive function. Nature Neurosci. 8, 79-84 (2005).

77. Lindersson, E. et al. Proteasomal inhibition by a-synuclein filaments and oligomers. J. Biol. Chem. 279, 12924-12934 (2004)

78. Gosavi, N., Lee, H. J., Lee, J. S., Patel, S. \& Lee, S. J. Golgi fragmentation occurs in the cells with prefibrillar $\alpha$-synuclein aggregates and precedes the formation of fibrillar inclusion. J. Biol. Chem. 277, 48984-48992 (2002).

79. Komatsu, M. et al. Loss of autophagy in the central nervous system causes neurodegeneration in mice. Nature 441, 880-884 (2006).

80. Hara, T. et al. Suppression of basal autophagy in neural cells causes neurodegenerative disease in mice. Nature 441, 885-889 (2006).

81. Kruger, R. et al. Familial parkinsonism with synuclein pathology: clinical and PET studies of A30P mutation carriers. Neurology 56, 1355-1362 (2001)

82. Schenk, D. et al. Immunization with amyloid- $\beta$ attenuates Alzheimer-disease-like pathology in the PDAPP mouse. Nature 400,173-177 (1999).

83. Janus, $C$. et al. $A \beta$ peptide immunization reduces behavioural impairment and plaques in a model of Alzheimer's disease. Nature 408, 979-982 (2000).

84. Bard, F. et al. Peripherally administered antibodies against amyloid $\beta$-peptide enter the central nervous system and reduce pathology in a mouse model of Alzheimer disease. Nature Med. 6, 916-919 (2000).

85. DeMattos, R. B. et al. Peripheral anti-A $\beta$ antibody alters CNS and plasma A $\beta$ clearance and decreases brain $A \beta$ burden in a mouse model of Alzheimer's disease. Proc. Natl Acad. Sci. USA 98, 8850-8855 (2001).

86. Gervais, F. et al. Targeting soluble A $\beta$ peptide with Tramiprosate for the treatment of brain amyloidosis. Neurobiol. Aging (in the press) published online 3 May 2006 (doi:10.1016/ j.neurobiolaging.2006.02.015).

87. McGeer, P. L., Schulzer, M. \& McGeer, E. G. Arthritis and anti-inflammatory agents as possible protective factors for Alzheimer's disease: a review of 17 epidemiologic studies. Neurology 47, 425-432 (1996).

88. Weggen, S. et al. A subset of NSAIDs lower amyloidogenic A $\beta 42$ independently of cyclooxygenase activity. Nature 414, 212-216 (2001)

89. Morihara, T., Chu, T., Ubeda, O., Beech, W. \& Cole, G. M. Selective inhibition of A $\beta 42$ production by NSAID R-enantiomers. J. Neurochem. 83, 1009-1012 (2002).

90. Eriksen, J. L. et al. NSAIDs and enantiomers of flurbiprofen target $\gamma$-secretase and lower A 42 in vivo. J. Clin. Invest. 112, 440-449 (2003).

91. Kopito, R. R. Aggresomes, inclusion bodies and protein aggregation. Trends Cell Biol. 10, 524-530 (2000).

Acknowledgements P.T.L. thanks the National Institutes of Health for supporting his work in this area. H.A.L. thanks R. Luthi-Carter, P. Fraering and N. Zawia for reviewing the manuscript, and the Swiss National Science Foundation for supporting research in his laboratory.

Author Information Reprints and permissions information is available at npg.nature.com/reprintsandpermissions. The authors declare competing financial interests: details accompany the paper at www.nature.com/nature. Correspondence should be addressed to P.T.L (lansbury@rics.bwh.harvard.edu) 